\title{
NOWHERE SIMPLICITY IN MATROIDS
}

\author{
R. DOWNEY ${ }^{\dagger}$
}

(Received 7 December 1981)

Communicated by J. N. Crossley

\begin{abstract}
We examine the concepts of nowhere simplicity in a wide class of abstract dependence systems. Initially we examine how many of the existing results valid for $L(\omega)$, the lattice of r.e. sets, have analogues valid for more general lattices. For example, we show that any $r$. e. subspace of $V_{\infty}$ can be decomposed into a pair of nowhere simple subspaces.

Later we begin an analysis of effective nowhere simplicity by giving a number of results which are new for both $L(\omega)$ and more general systems. In particular we examine and define the concept of being a member of a maximal pair. We prove:
\end{abstract}

THEOREM. An r.e. (closed) set A may be decomposed into an r.e. maximal pair if and only if $A$ is simple.

COROLlARY. There exist effectively nowhere simple (closed) sets in each r. e. degree $(\neq 0)$.

1980 Mathematics subject classification (Amer. Math. Soc.): primary 03 D 45; secondary 03 D 25, 03 G 10.

\section{Introduction}

The study of constructivity in mathematics seems to proceed in two stages. The first stage is to identify those processes or functions which we shall call effective. Having identified these, we may then proceed to the second stage, which is to analyse the "effective content" of various mathematical systems. Typically, we identify the class of effective functions with recursive functions and this we shall

\footnotetext{
† These results were obtained whilst the author was at Monash University, and form part of his Ph.D. thesis.

(c) Copyright Australian Mathematical Society 1983
} 
do here. This (admittedly vague) description of the second stage is perhaps best clarified by an example (essentially Metakides and Nerode [7]). Consider the classical theorem "Every vector space has a basis". We wish to analyse the constructive content of this theorem. To clarify the situation we ask that our spaces be recursive sets (identified with $\omega$ ), the underlying field be a recursive field, the operations of scalar multiplication and vector addition be recursive functions and identity be a recursive relation. We call such a vector space $V$ recursively presented. (If identity is only r.e., we say $V$ is r.e.presented.)

Question. Does every recursively presented vector space have a recursive basis? Answer. NO.

In essence this shows that the classical theorem has a nonconstructive element. Of course, one important area of development is really devoted to the first stage, that is, to the analysis of the structure of the set of those functions which we call effective. Post initiated the study of the lattice $L(\omega)$ of recursively enumerable (r.e.) sets. One important insight due to Metakides and Nerode [7] is that questions like the one above can often be answered by an analysis of $L\left(V_{\infty}\right)$, the lattice of r.e. subspaces of a fully effective vector space $V_{\infty}$. By fully effective we mean that $V_{\infty}$ is recursively presented and has a dependence algorithm, that is, given $a, b_{1}, \ldots, b_{n} \in V_{\infty}$ we can effectively determine whether or not $a \in$ $\left(b_{1}, \ldots, b_{n}\right)^{*}$, where $A^{*}$ denotes the subspace generated by $A$. To see this, we list a few observations. The first observation is that every r.e. (recursively) presented vector space $Q$ is recursively isomorphic to $V_{\infty} \bmod W$ for some r.e. (recursive) subspace $W$ of $V_{\infty}$. The second observation is that an r.e. vector space $Q$ has a dependence algorithm if and only if $Q$ has a recursive basis. The third observation is that $V_{\infty} \bmod W$ has a recursive basis if and only if $W$ is complemented in $L\left(V_{\infty}\right)$. Thus to obtain the negative answer to the earlier question we simply need to construct a recursive subspace of $V_{\infty}$ which is not complemented in $L\left(V_{\infty}\right)$.

This form of analysis has been generalized in two ways. One way is to consider more general mathematical systems. In this paper we consider one such generalization to abstract dependence (Steinitz) systems (see Section 1), others are to topology (Kalantari, Retzlaff, Leggett, Remmel), abelian groups (Lin, Smith), fields (Metakides and Nerode), orderings (Crossley, Manaster, Remmel) Boolean algebras (La Roche, Remmel) and many other systems. The other form of exploration is a more intensive study of $L\left(V_{\infty}\right)$ (for example, Baldwin, Shore and most of the earlier authors).

One important point of analysing $L\left(V_{\infty}\right)$ (and other areas) is perhaps worth mentioning, namely, such analyses shed a great deal of light on $L(\omega)$. One example is Metakides and Nerode's adaptation of an $e$-state construction from 
$L(\omega)$ to $L\left(V_{\infty}\right)$. The key aspect in both lattices is the interplay of dependence and recursion theory which makes the adaptation possible.

The main thrust of much of the analysis of $L\left(V_{\infty}\right)$ has been in constructing r.e. subspaces $W$ of $V_{\infty}$ whose complements contain few r.e. subspaces. One powerful example of this is the construction of a supermaximal subspace of $V_{\infty}$. We say $W \in L\left(V_{\infty}\right)$ is supermaximal if $\operatorname{dim}\left(V_{\infty} / W\right)=\infty$ and, for all $Q \in L\left(V_{\infty}\right)$, if $Q \supset W$ then either $Q=V_{\infty}$ or $\operatorname{dim}(Q / W)<\infty$. Note that the existence (proved in [8]) of an r.e. supermaximal subspace of $V_{\infty}$ implies that there exists an r.e. (recursively) presented vector space whose only r.e. subspaces are finite dimensional.

In this paper, we concentrate upon the other side of things, that is, upon r.e. subspaces where complements contain many r.e. subspaces. Their behaviour differs from their analogues in $L(\omega)$. For example, the natural analogue of a recursive set that is a decidable (complemented) subspace $V_{\infty}$ of $V_{\infty}$. It is interesting that even the behaviour of decidable subspaces of $V_{\infty}$ differs markedly from the corresponding behaviour of recursive subsets of $\omega$. In particular, C. J. Ash and the author [0] have shown that every r.e. subspace is the direct sum of a pair of decidable subspaces. Here we concentrate upon nowhere simplicity and effective nowhere simplicity in $L\left(V_{\infty}\right)$ (more generally $L(U)$ ) as defined in Section 1. Briefly, a set (subspace) $V$ is nowhere simple if every r.e. set (subspace) containing $V$ contains an r.e. infinite (infinite dimensional) subset (subspace) disjoint from $V$. Thus the existence of a nowhere simple nondecidable subspace establishes the existence of a represented vector space without a recursive basis, yet having many r.e. subspaces (of infinite dimension).

Rather than listing our results we close with a couple of comments. Our initial results are adaptations of $L(\omega)$ constructions and are therefore only new in terms of their algebraic settings. However this is interesting because (a) results concerning $L(\omega)$ do not always adapt to $L\left(V_{\infty}\right)$ and (b) the results concerning automorphisms of $L(\omega)$ and $L\left(V_{\infty}\right)$, which we obtain, simultaneously concern themselves with two entirely different objects for there are $2^{\kappa_{0}}$ automorphisms of $L(\omega)$ and only $\boldsymbol{\kappa}_{0}$ of $L\left(V_{\infty}\right)$ (all essentially effective).

Finally all of our later results (for example, Proposition 1.15, Corollary 1.16) are new for $L(\omega)$ and hence explore the notion of an (effectively) nowhere simple set which is of considerable importance in $L(\omega)$.

This paper extends some of the investigations to be found in Metakides and Nerode [8], and the author [3] and [4]. We shall use the basic results and definitions of [8]. The structures we are interested in are a subclass of the class of Steinitz (closure) systems.

Definition 1.0 ([8]). A Steinitz (closure) system $(U, \mathrm{cl})$ consists of a set $U$ and an operation cl: $P(U) \rightarrow P(U)$ such that for all subsets $A$ and $B$ of $U$ 
(i) $A \subset \operatorname{cl}(A)$,

(ii) $A \subset B$ implies $\operatorname{cl}(A) \subset \mathrm{cl}(B)$,

(iii) $\operatorname{cl}(\operatorname{cl}(A))=\operatorname{cl}(A)$,

(iv) $x \in \operatorname{cl}(A)$ implies that, for some finite $A^{\prime} \subset A, x \in \operatorname{cl}\left(A^{\prime}\right)$,

(v) (exchange) $x \in \operatorname{cl}(A \cup\{y\})-\operatorname{cl}(A)$ implies $y \in \operatorname{cl}(A \cup\{x\})$.

A Steinitz system $(U, \mathrm{cl})$ has recursive dependence if $U$ is recursive and there is a uniformly effective procedure which, when applied to elements $a, b_{1}, \ldots, b_{n}$ of $U$ determines in a finite number of steps whether or not $a \in \operatorname{cl}\left(b_{1}, \ldots, b_{n}\right)$.

EXAMPles of Steinitz Systems. (i) $(\omega, \mathrm{cl})$. Here $\omega$ is the integers and $\operatorname{cl}(A)=A$.

(ii) $\left(V_{\infty},{ }^{*}\right)$. Again, $V_{\infty}$ denotes a fully effective vector space with $A^{*}$ the subspace generated by $A$ (see $[7,8])$.

(iii) $\left(F_{\infty}, \mathrm{cl}\right)$. Here $F_{\infty}$ denotes a fully effective algebraically closed field (that is, with an infinite recursive transcendence base) and cl denotes an effective algebraic closure operator (see [8]).

(iv) $\left(V_{\infty}(F), \mathrm{Kl}\right)$. We say that $x \in V_{\infty}$ depends affinely on $y_{1}, \ldots, y_{n}$ in $V_{\infty}$ if there exists $\lambda_{1}, \ldots, \lambda_{n} \in F$ such that $x=\Sigma \lambda_{i} y_{i}$ and $\Sigma \lambda_{i}=1$. If $x$ depends affinely on $y_{1}, \ldots, y_{n}$ we write $x \in \operatorname{Kl}\left(y_{1}, \ldots, y_{n}\right)$. This is the well-known generalization of occurence on points, lines, planes (see [4]).

(v) Let $V$ be a recursive subset of $V_{\infty}$. Define $(V$,cl) via (for $A \subset V$ ), $\operatorname{cl}(A)=A^{*} \cap V$. This is wide class oftentimes referred to as $V^{*}$-algebras (see [10]).

If the reader is unfamiliar with Steinitz systems he is asked to keep $(\omega, \mathrm{cl})$ and $\left(V_{\infty},{ }^{*}\right)$ in mind. We give a brief review of some of the basic results from [8] and refer the reader there for further details:

We say a set $A$ is independent (over $B$ ) if $A \neq 0$ and for all $a \in B, a \notin \mathrm{cl}_{B}(A-$ $\{a\})$ where $\operatorname{cl}_{B}(X)=\operatorname{cl}(B \cup X)$. Similarly we may define spanning sets, bases and the like. We may write $A+B$ for $\operatorname{cl}(A \cup B)$. A pair of closed sets $A, B$ are independent if each basis of $A$ is independent over $B$ and conversely. Akin to $L\left(V_{\infty}\right)$ we let $L(U)$ denote the lattice of r.e. closed subsets of $U$.

The following result shows how Steinitz systems are similar to $V_{\infty}$ :

Proposition 1.1. For $A, B$ subsets of $U$ in a Steinitz closure system $(U, \mathrm{cl})$,

(i) if $A$ is closed, $I$ is independent, $S$ spans $A$ and $I \subseteq S$, then there is as basis $X$ of $A$ extending $I$ with $I \subseteq X \subseteq S$.

(ii) Let $A$ and $B$ be closed and $B \subset A$. Suppose $B_{1}$ is a basis for $B$ and $A_{1}$ a basis for $A$ over $B$. Then $A_{1} \cup B_{1}$ is a basis for $A$.

(iii) Let $X \cup Y$ be independent (over $B$ ). Then $\mathrm{cl}_{B}(X \cap Y)=\mathrm{cl}_{B}(X) \cap \mathrm{cl}_{B}(Y)$.

(iv) If $B$ is closed, $I$ is independent over $B$ and $x \in \mathrm{cl}_{B}(I)$, then there is a smallest finite set $I^{\prime} \subset I$ with $x \in \mathrm{cl}_{B}\left(I^{\prime}\right)$. 
Proof. For (i) see [8], for (ii) see [8], Theorem 2.4, for (iii) see 2.6 of [8] and (iv) is 2.7 of [8].

The (unique) smallest finite subset $I^{\prime}$ of $I$ with $x \in \mathrm{cl}_{B}\left(I^{\prime}\right)$ in (iv) of 2.3 is referred to as the support of $x$ over $B$ relative to $I$ and is denoted by $\operatorname{supp}_{I}(x)$ (over $B$ ). As in [8] the dimension of $A$ over $B(\operatorname{dim}(A / B))$ is the cardinality of any basis for $A$ over $B$. If $(U, \mathrm{cl})$ has recursive dependence we can determine effectively for a finite $A$ and $B$ whether or not $\operatorname{cl}(A) \subset \operatorname{cl}(B), A$ is independent over $B$, and for any $Q \in L(U)$ find an r.e. basis of $Q$ (extending any given finite independent subset). Finally for $V \in L(U)$, the dependence set $D(V)$ if $V$ in $L(U)$ is $U_{k \in W} D(V)_{k}$ where $D(V)_{k}$ is the set of all $k$-tuples dependent over $V$. The dependence degree is the (Turing) degree of $D(V)$ of $D(V) \equiv_{T}$ o we say $V$ is decidable. Note that $V$ is decidable if and only if $V$ is complemented in $L(U)$.

Definition 1.2. Let $(U, \mathrm{cl})$ be a Steinitz system. We say $(U, \mathrm{cl})$ has C.I.P. (closure intersection property) if, for all closed subsets $D$ of $U$, whenever $A$ and $B$ are both independent over $D$ and $\operatorname{cl}_{D}(A) \cap \mathrm{cl}_{D}(B)=\operatorname{cl}(D)$, then $A \cup B$ is independent over $D$.

Not every Steinitz system has C.I.P.; of our examples, $\left(F_{\infty}, \mathrm{cl}\right)$ and $\left(V_{\infty}, \mathrm{Kl}\right)$ do not. The C.I.P. was introduced in [3] mainly to account for a certain class of phenomena which occur in $L\left(V_{\infty}\right)$ but not $L\left(F_{\infty}\right)$. Henceforth $(U$, cl) will always denote a Steinitz closure system with recursive dependence and with C.I.P. As an analogue of the Shore's definition ([14], page 323) we have:

Definition 1.3. Let $S \in L(U)$ with $\operatorname{dim}(U / S)=\infty$. We say $S$ is nowhere simple if, whenever $Q \in L(U)$ with $Q \supset S$ and $\operatorname{dim}(Q / S)=\infty$, there exists an r.e. $Q^{\prime} \subset Q$ such that $\operatorname{dim}\left(Q^{\prime}\right)=\infty$ and $Q^{\prime} \cap S=\operatorname{cl}(\varnothing)$.

It has been suggested ${ }^{\dagger}$ that there is a stronger notion of nowhere simplicity. Namely we say $V$ is strongly nowhere simple if, for all $Q \in L(U)$ with $\operatorname{dim}(Q / V)$ $=\infty$ (that is $\operatorname{dim}(Q+V / V)=\infty)$ there exists $Q^{\prime} \subset Q$ such that $\operatorname{dim}\left(R^{\prime}\right)=\infty$ and $Q^{\prime} \cap V=\{0\}$. However we may show they are equivalent as follows:

Proposition. Suppose $V \in L(U)$ and $(U, \mathrm{cl})$ has C.I.P. Then $V$ is strongly nowhere simple $\leftrightarrow V$ is nowhere simple.

Proof. $(\rightarrow)$ is clear.

$(\leftarrow)$ Suppose $V$ is nowhere simple and that $Q \in L(U)$ with $\operatorname{dim}(Q / V)=\infty$. As $V$ is nowhere simple there exists $Q^{\prime} \subset Q+V$ such that $Q^{\prime} \cap V=\operatorname{cl}(\varnothing)$ and

\footnotetext{
'It was observed by the referee of a paper (by the author) "Co-immune subspaces and complementations in $V_{\infty}$," (to appear J. Symbolic Logic) that this form was needed for some applications.
} 
$\operatorname{dim}\left(Q^{\prime}\right)=\infty$. Compute a recursive basis $B$ of $Q+V$ as follows. Let $\left\{q_{1}, q_{1}, q_{2}, \ldots\right\}$ be a recursive basis of $Q$. Let $\left\{v_{0}, v_{1}, \ldots\right\}$ be a recursive basis of $V$. Define $B=\cup_{s} B_{s}$ in stages:

Stage 0. $B_{0}=\left\{q_{1}\right\}$.

Stage 1.

$$
B_{1}= \begin{cases}B_{0} \cup\left\{v_{0}\right\} & \text { if } B_{0} \cup\left\{v_{0}\right\} \text { is independent, } \\ B_{0} & \text { otherwise. }\end{cases}
$$

Stage $2 s(s>0)$.

$$
B_{2 s}= \begin{cases}B_{2 s-1} \cup\left\{q_{s}\right\} & \text { if } B_{2 s-1} \cup\left\{q_{s}\right\} \text { independent, } \\ B_{2 s-1} & \text { otherwise. }\end{cases}
$$

Stage $2 s+1$.

$$
B_{2 s+1}= \begin{cases}B_{2 s} \cup\left\{v_{s}\right\} & \text { if } B_{2 s} \cup\left\{v_{s}\right\} \text { independent, } \\ B_{2 s} & \text { otherwise. }\end{cases}
$$

Set $B=\cup_{s} B_{s}$ and let $B=\left\{b_{0}, b_{1}, \ldots\right\}$ list the elements of $B$.

Let $\left\{z_{0}, z_{1}, \ldots\right\}$ be a recursive basis of $Q^{\prime}$. Note that

$$
\text { each } b_{i} \text { is either a } q_{i} \text { or a } v_{i} \text {. }
$$

Compute $\operatorname{supp}\left(z_{i}\right)=\left\{b_{i_{0}}, \ldots, b_{i_{n}}\right\}$ and by $(1), \operatorname{supp}\left(z_{i}\right)=\left\{q_{i_{0}} \cdots q_{i_{k}}, v_{i_{0}} \cdots v_{i_{t}}\right\}$. We claim:

(*) there exist $Q_{i} \in \operatorname{cl}\left(q_{i_{0}}, \ldots, q_{i_{k}}\right)$ with $q_{i} \notin V$ and $V_{i} \in \operatorname{cl}\left(v_{i_{0}}, \ldots, v_{i_{r}}\right)$ such that $z_{i} \in \operatorname{cl}\left(Q_{i}, V_{i}\right)$.

We prove this by induction. We only do the $Q_{i}$ case, the $V_{i}$ case is similar. If $k=1$, then $\operatorname{supp}\left(z_{i}\right)=\left\{q_{i_{0}}, v_{i_{0}}, \ldots, v_{i_{t}}\right\}$. Now, by C.I.P. $\operatorname{cl}\left(z_{i}, q_{i_{0}}\right) \cap \operatorname{cl}\left(v_{i_{0}}, \ldots, v_{i_{t}}\right)$ $\neq \operatorname{cl}(\varnothing)$ (else, $\left\{z_{i}, q_{i_{0}}, \ldots, v_{i}\right\}$ is independent). Find $\eta \notin \operatorname{cl}(\varnothing)$ and $\eta \in \operatorname{cl}\left(z_{i}, q_{i}\right)$ $\cap \operatorname{cl}\left(v_{i_{0}}, \ldots, v_{i_{r}}\right)$. By exchange, it follows that:

$$
z_{i} \in \operatorname{cl}\left(q_{i_{0}}, \eta\right)
$$

Consider $k=m+1 . \operatorname{supp}\left(z_{i}\right)=\left\{q_{i_{0}}, \ldots, q_{i_{m+1}}, v_{i_{0}}, \ldots, v_{i_{i}}\right\}$, and so by (2) (with similar manipulations) $z_{i} \in \operatorname{cl}\left(q_{i_{0}}, \eta\right)$ where $\eta \in \operatorname{cl}\left(q_{i_{1}}, \ldots, q_{i_{m+1}}, v_{i_{0}}, \ldots, v_{i_{t}}\right)$. Without loss of generality $\operatorname{supp}(\eta)=\left\{q_{i_{1}}, \ldots, q_{i_{m+1}}, v_{i_{0}}, \ldots, v_{i_{t}}\right\}$. By induction there exist $\gamma_{i}, V_{i}$ such that $\eta \in \operatorname{cl}\left(\gamma_{i}, V_{i}\right)$ with $\gamma_{i} \in \operatorname{cl}\left(q_{i}, \ldots, q_{i_{m+1}}\right)$ and $V_{i} \in$ $\operatorname{cl}\left(v_{i_{0}}, \ldots, v_{i_{i}}\right)$, so that $z_{i} \in \operatorname{cl}\left(q_{i_{0}}, \gamma_{i}, V_{i}\right)$. By (2) again, there exists $Q_{i}$ such that $Q_{i} \in \operatorname{cl}\left(q_{i_{0}}, \ldots, q_{i_{m+1}}\right)$ with $z_{i} \in\left(Q_{i}, V_{i}\right)$ and the claim follows as $Q_{i} \notin V$ else $z_{i} \in V$.

Set $T=\left\{Q_{i} \mid i \in \omega\right\}$. It is easy to show that $\operatorname{cl}(T) \cap V=\operatorname{cl}(\varnothing)$ since $T$ is independent over $V$. This is because $\forall n \in \omega\left(\operatorname{cl}_{V}\left(z_{1}, \ldots, z_{n}\right)=\operatorname{cl}_{V}\left(Q_{1}, \ldots, Q_{n}\right)\right)$ and so $\left\{Q_{i} \mid i \in \omega\right\}$ is independent over $V$. By C.I.P., $\operatorname{cl}\left\{Q_{i} \mid i \in \omega\right\} \cap V=\operatorname{cl}(\varnothing)$. 
Thus, we have constructed an r.e. infinite dimension closed subset (namely $\operatorname{cl}(T))$ such that $\operatorname{cl}(T) \subset Q$ and $\operatorname{cl}(T) \cap V=\operatorname{cl}(\varnothing)$. Thus $V$ is strongly nowhere simple.

REMARK. If $(V, \mathrm{cl})$ does not have C.I.P. this equivalence is not clear. In fact we believe that it does not hold, but do not pursue strong nowhere simplicity further.

The reason for considering systems with C.I.P. becomes clear if one takes an example of a Steinitz system without C.I.P. Let $Q \in L\left(F_{\infty}\right)$. Suppose $M \supsetneqq Q \not$ for some $M \in L\left(F_{\infty}\right)$. Let $b \in M-Q$. Let $\left\{q_{1}, q_{2}, \ldots\right\}$ denote a recursive basis of $Q$.

Let $R=\left\{b+q_{1}, b^{2}+q_{2}, b^{3}+q_{3}, \ldots\right\}$. Now $\operatorname{dim}(\operatorname{cl}(R))=\infty, \operatorname{cl}(R) \subset M$ and $\operatorname{cl}(R) \cap Q=\operatorname{cl}(\varnothing)$. Thus in $\left(F_{\infty}, \mathrm{cl}\right)$ every closed subset is nowhere simple. Similar problems occur if one tries to define simplicity in $\left(F_{\infty}, \mathrm{cl}\right)$. This was discussed in [4]. Recall that $Q \in L(V)$ is simple if for all $R \in L(V)$ if $R \cap Q=$ $\operatorname{cl}(\varnothing)$ then $\operatorname{dim}(R)<\infty$. In systems with C.I.P. there are simple closed subsets. Thus there are many elements of $L(U)$ that are not nowhere simple.

Proposition 1.4. Let $Q \in L(U)$. Then there exists $Q_{1}, Q_{2} \in L(U)$ with $Q_{1}$ and $Q_{2}$ independent. $Q_{1}+Q_{2}=\operatorname{cl}\left(Q_{1} \cup Q_{2}\right)=Q$ and $Q_{1}, Q_{2}$ are nowhere simple.

Proof. We build $A_{i}=\cup_{s} A_{i}^{s}$ in stages (for $i=1,2$ ) so that $A_{1} \cap A_{2}=\varnothing$ and $A_{1} \cup A_{2}$ is a basis of $Q$. At each stage $s, \operatorname{cl}\left(A_{1}^{s} \cup A_{2}^{s}\right)=\operatorname{cl}\left(Q^{s}\right)$. Clearly we may assume $\operatorname{dim}(Q)=\infty$. Let $W_{e}$ enumerate the $e$ th r.e. closed subset of $U$. We attempt to meet the following requirements (for $i=1,2) P_{\langle e, n)}^{i}: \operatorname{dim}\left(W_{e} \cap \operatorname{cl}\left(A_{i}\right)\right.$ ) $\geqslant n$. The priority ranking is $P_{0}^{1}, P_{0}^{2}, P_{1}^{1}, P_{1}^{2}, P_{2}^{1}, \ldots$

Definition 1.4.1. $P_{\langle e, n\rangle}^{i}$ is satisfied at stage $s$ if $\operatorname{dim}\left(\operatorname{cl}\left(W_{e}^{s}\right) \cap \operatorname{cl}\left(A_{i}^{s}\right)\right) \geqslant n$.

Definition 1.4.2. $P_{\langle e, n\rangle}^{i}$ requires attention at stage $s+1$ if $P_{\langle e, n\rangle}^{i}$ is the unsatisfied requirement of highest priority such that

$$
\exists \psi \in \operatorname{cl}\left(W_{e}^{s+1}\right) \cap \operatorname{cl}\left(Q^{s+1}\right) \text { with } \psi \notin \operatorname{cl}\left(A_{1}^{s} \cup A_{2}^{s}\right) .
$$

Construction. Stage $0 . A_{1}^{0}=A_{2}^{0}=\varnothing$.

Stage $s+1$. If $\operatorname{cl}\left(Q^{s+1}\right)=\operatorname{cl}\left(Q^{s}\right)$ do nothing. If $\operatorname{cl}\left(Q^{s+1}\right) \neq \operatorname{cl}\left(Q^{s}\right)$ find the requirement $P_{\langle e, n\rangle}^{i}$ which requires attention at stage $s+1$. Let $\psi$ be least for $\langle e, n\rangle$ and set

$$
A_{j}^{s+1}= \begin{cases}A_{j}^{s} & \text { if } j \neq 1, \\ A_{i}^{s} \cup\{\psi\} & \text { if } j=i .\end{cases}
$$

Let $A_{i}=\cup_{s} A_{i}^{s}$ and $Q_{i}=\operatorname{cl}\left(A_{i}\right)$ for $i=1,2$.

End of construction. 
We claim that both $Q_{1}$ and $Q_{2}$ are nowhere simple. Suppose $Q_{1}$ is not. Then (*) below holds.

(*) there exists $W_{e} \supset A_{1}$ with $W_{e}$ containing no infinite dimensional r.e. closed subset meeting $\mathrm{cl}\left(A_{1}\right)$ only trivially.

Let

$$
Z=\left\{x \mid \exists s, t\left(t>s \& x \in \operatorname{cl}\left(W_{e}^{s}\right) \& x \notin \operatorname{cl}\left(Q^{s}\right) \& x \in \operatorname{cl}\left(Q^{t}\right)\right)\right\} .
$$

Suppose $Z$ is infinite. Then it is easy to see that $\forall n\left(P_{\langle e, n\rangle}^{i}\right.$ is met (for $\left.i=1,2\right)$ ), and therefore $\operatorname{dim}\left(W_{e} \cap A_{2}\right)=\infty$ giving a contradiction to (*). Thus $Z$ is finite. Hence there is a stage $s_{0}$ such that

$$
\forall s>s_{0}\left(x \in \operatorname{cl}\left(W_{e}^{s}\right) \Rightarrow x \in \operatorname{cl}\left(Q^{s}\right) \vee \forall t>s\left(x \notin \operatorname{cl}\left(Q^{t}\right)\right)\right) .
$$

Now if $\operatorname{dim}\left(\operatorname{cl}_{Q}\left(W_{e}\right) / Q\right)<\infty$, then as $\left(U\right.$, cl) has C.I.P. it follows that $\operatorname{dim}\left(W_{e} \cap\right.$ $\left.\operatorname{cl}\left(A_{2}\right)\right)<\infty$. For let $\operatorname{dim}\left(\operatorname{cl}_{Q}\left(W_{e}\right) / Q\right)<\infty$ and suppose $\operatorname{dim}\left(W_{e} \cap \operatorname{cl}\left(A_{2}\right)\right)<\infty$. Let $B_{1}$ be a basis of $W_{e}$ over $\operatorname{cl}\left(A_{1} \cup\left(W_{e} \cap A_{2}\right)\right)$, and $B_{2}$ a basis of $\operatorname{cl}(Q)$ over $\operatorname{cl}\left(A_{1} \cup\left(W_{e} \cap A_{2}\right)\right)=T$. Then $B_{1} \cap B_{2}=\varnothing$ and $\operatorname{cl}_{T}\left(B_{1}\right) \cap \mathrm{cl}_{T}\left(B_{2}\right)=\operatorname{cl}(T)$. Therefore $B_{1} \cup B_{2}$ is independent over $T$ and so $B_{1}$ is independent over $\operatorname{cl}\left(B_{2} \cup\right.$ $\left.\operatorname{cl}\left(A_{1} \cup\left(W_{e} \cap A_{2}\right)\right)\right)=Q$. This implies that $\operatorname{dim}\left(\operatorname{cl} Q\left(W_{e}\right) / Q\right)=\infty-\mathrm{a}$ contradiction.

Thus without loss of generality we may suppose

$$
\operatorname{dim}\left(\mathrm{cl}_{Q}\left(W_{e}\right) / Q\right)=\infty \text {. }
$$

Now under the assumption (4) we show that (3) implies that we can produce an r.e. subset $R$ of $W_{e}$ with $\operatorname{cl}(R) \cap Q=\operatorname{cl}(\varnothing)$ and $R$ independent over $\operatorname{cl}(Q)$.

Construction. Stage 0 . Let $x_{0}$ be the first $x$ occurring at the least stage $s^{0}>s_{0}$ such that $x \in \operatorname{cl}\left(W_{e}^{s^{0}}\right)-\operatorname{cl}\left(Q^{s_{0}}\right)$. Let $R^{0}=\left\{x_{0}\right\}$.

Stage $t+1$. Let $R^{t}=\left\{x_{0}, \ldots, x_{t}\right\}$ and suppose we have defined $s^{t}>\cdots>s^{0}$. Let $x_{t+1}$ be the first $x$ occurring at the least stage $s^{t+1}>s^{t}$ such that $x_{t+1} \in$ $\operatorname{cl}\left(W_{e}^{s^{t+1}}\right)-\operatorname{cl}\left(Q^{s^{t+1}}\right)$ and $x_{t+1} \notin \operatorname{cl}\left(Q^{s^{t+1}} \cup\left\{x_{0}, \ldots, x_{t}\right\}\right)$. Let $R=\cup_{t} R^{t}$ and $J=\operatorname{cl}(R)$.

End of construction.

Note that by (4) $\operatorname{card}(R)=\infty$. We claim $R$ is independent over $Q$. For suppose $\left\{x_{0}, \ldots, x_{t}\right\}$ is independent over $Q$ but $\left\{x_{0}, \ldots, x_{t+1}\right\}$ is dependent over $Q$. Now, by construction $\left\{x_{0}, \ldots, x_{t+1}\right\}$ independent over $\operatorname{cl}\left(Q^{s^{t+1}}\right)$. Thus at some stage $z>s^{t+1},\left\{x_{0}, \ldots, x_{t+1}\right\}$ dependent over $\operatorname{cl}\left(Q^{z}\right)$ but independent over $\operatorname{cl}\left(Q^{z-1}\right)$. Let $T$ be a basis of $\operatorname{cl}\left(Q^{z-1}\right)$ and $T \cup\{\psi\}$ a basis of $\operatorname{cl}\left(Q^{z}\right)$. By C.I.P. it follows that $\operatorname{cl}(T \cup\{\psi\}) \cap \operatorname{cl}\left\{x_{0}, \ldots, x_{t+1}\right\} \neq \operatorname{cl}(\varnothing)$. Thus as $\operatorname{cl}\left(\left\{x_{0}, \ldots, x_{t+1}\right\}\right) \subset$ $\operatorname{cl}\left(W_{e}^{s^{s+1}}\right)$ and $z>s^{t+1}$ and $\operatorname{cl}(T \cup\{\psi\}) \subset \operatorname{cl}\left(Q^{z}\right)$ it follows that

$$
\exists \eta\left(\eta \in \operatorname{cl}\left(W_{e}^{s^{t+1}}\right) \& \eta \notin \operatorname{cl}\left(Q^{s^{t+1}}\right) \& \exists z>s^{t+1}\left(\eta \in \operatorname{cl}\left(Q^{z}\right)\right)\right) .
$$


However by construction $s^{t+1}>s_{0}$ and this contradicts (3). Thus $R$ is independent over $Q$ and the result follows.

As Shore pointed out in [14] for $L(\omega)$ we can control the degrees of $Q_{1}$ and $Q_{2}$ to ensure both $Q_{1}$ and $Q_{2}$ are r.e. nonrecursive if $Q$ is r.e. nonrecursive. Recall a decidable closed subset is one with an r.e. independent complement. If we use the method of either Shore [15] or Retzlaff [11] we can ensure similarly that if $Q$ is not complemented, $Q_{1}$ and $Q_{2}$ are not complemented.

Propositron 1.5. Let $Q \in L(U)$, with $Q$ not complemented in $L(U)$. Then there exist noncomplemented $Q_{1}, Q_{2} \in L(U)$ with $\mathrm{cl}\left(Q_{1} \cup Q_{2}\right)=Q, Q_{1}$ and $Q_{2}$ independent and both $Q_{1}$ and $Q_{2}$ nowhere simple.

In any case, each decidable closed subset of $U$ is expressible as the intersection of two noncomplemented members of $L(U)$. Thus

COROLlary 1.8. The r.e. noncomplemented nowhere simple closed subsets of $U$ generate $L(U)$ (under $\cap$ and + ).

In $L(U)$ we define $A={ }^{*} B$ if and only if there exists a finite $F \subset U$ such that

$$
\operatorname{cl}(A \cup F)=\operatorname{cl}(B \cup F) \text {. }
$$

Clearly $=^{*}$ is an equivalence relation. With this, we define the lattice $L^{*}(U)$ of members of $U$ modulo the equivalence $=*$. It is not too difficult to obtain the following, which is an analogue of Shore's automorphism result for $L^{*}(\omega)$.

Corollary 1.9. If $\varphi: L^{*}\left(V_{\infty}\right) \rightarrow L^{*}\left(V_{\infty}\right)$ is any elementary monomorphism and $\varphi$ is the identity on $S$, a nontrivial class of r.e. subspaces closed under recursive isomorphism. Then $\varphi$ is the identity on $L^{*}\left(V_{\infty}\right)$.

COROLlarY 1.10. If $\varphi_{1}$ and $\varphi_{2}$ are automorphisms of $L^{*}\left(V_{\infty}\right)$ agreeing on $S$ then $\varphi_{1}=\varphi_{2}$.

In [14] Shore goes on to prove as corollaries many results concerning extendability and nonextendability of automorphisms of subclasses of $L(\omega)$. In general, analogues of his results do not seem to hold. For example Lemma 7 of [13] does not hold in $L\left(V_{\infty}\right)$. There are many reasons for this. In his thesis Guichard has shown that there are only $\boldsymbol{\aleph}_{0}$ automorphisms of $L\left(V_{\infty}\right)$, each induced by a recursive semilinear transformation. Thus the automorphisms of $L\left(V_{\infty}\right)$ are very different from those of $L(\omega)$. 
One method of producing nowhere simple sets in $L(\omega)$ is to split a maximal set $M$ into two distinct r.e. non-recursive sets $M_{1}$ and $M_{2}$. Now $M_{1}$ and $M_{2}$ are both effectively nowhere simple in the sense of the following definition, which is the analogue of Shore's definition for $L(\omega)$ in [14].

Definition 1.10. Let $S \in L(U)$. We says $S$ is effectively nowhere simple if $S$ is nowhere simple and whenever $Q \supset S, \operatorname{dim}(Q / S)=\infty$ and $Q \in L(U)$ we can effectively compute $Q^{\prime} \subset Q$ such that $Q^{\prime} \in L(U), \operatorname{dim}\left(Q^{\prime}\right)=\infty$ and $Q^{\prime} \cap S=$ $\operatorname{cl}(\varnothing)$.

In fact as we found in [5] the sets $M_{1}$ and $M_{2}$ form a maximal pair where we defined $M_{1}$ and $M_{2} \in L(\omega)$ to be a maximal pair if, for all $C \in L(\omega)$ with $C \supset M_{1}$ (resp. $\left.C \supset M_{2}\right)$ and $C \cap M_{2}=\varnothing$ (resp. $\left.C \cap M_{1}=\varnothing\right), \operatorname{card}\left(C-M_{1}\right)$ $<\infty\left(\right.$ resp. $\left.\operatorname{card}\left(C-M_{2}\right)<\infty\right)$. It is easy to see that any member of a maximal pair is effectively nowhere simple. In $L(U)$ we have:

Definition 1.11. Let $A_{1}, A_{2} \in L(U)$. We say $A_{1}$ and $A_{2}$ are a maximal pair if $\operatorname{dim}\left(U / A_{1}+A_{2}\right)=\infty, A_{1} \cap A_{2}=\operatorname{cl}(\varnothing)$, and whenever $C \in L(U)$ with $C \supset A_{1}$ (resp. $\left.C \supset A_{2}\right)$ and $C \cap A_{2}=\operatorname{cl}(\varnothing)$ (resp. $C \cap A_{1}=\operatorname{cl}(\varnothing)$ ), $\operatorname{dim}\left(C / A_{1}\right)<\infty$ (resp. $\left.\operatorname{dim}\left(C / A_{2}\right)<\infty\right)$.

In a Steinitz system with the C.I.P. there is a very easy way of producing maximal pairs. We say $Q \in L(U)$ is maximal if $\operatorname{dim}(U / Q)=\infty$ and, for all $W \in L(U)$ if $W \supset Q$ either $\operatorname{dim}(U / W)<\infty$ or $\operatorname{dim}(W / Q)<\infty$.

Proposition 1.12. Let $M$ be any maximal closed subset of $U$ and let $\left(M_{1}+M_{2}\right)$ $=M$ be any r.e. nondecidable disjoint decomposition of $M$. Then $\left(M_{1}, M_{2}\right)$ is a maximal pair.

Proof. Let $C \in L(U)$ with $\operatorname{dim}\left(C / M_{1}\right)=\infty, C \supset M_{1}$ and $C \cap M_{2}=\operatorname{cl}(\varnothing)$. Now $\operatorname{dim}\left(\operatorname{cl}\left(C \cup M_{2}\right) / \operatorname{cl}\left(M_{1} \cup M_{2}\right)\right)=\infty$ (by C.I.P.) and so $\operatorname{dim}(U / \operatorname{cl}(C \cup$ $\left.\left.M_{2}\right)\right)<\infty$. Thus both $C$ and $M_{2}$ are decidable closed subsets contradicting the choice of $M_{2}$.

We note that as the author (paper in preparation) has shown that any r.e. nondecidable subspace may be decomposed into the direct sum of a pair of subspaces no r.e. basis of either of which may be extended to an r.e. basis of $V_{\infty}$, there exists a nowhere simple subspace no basis of which is fully extendable.

This construction allows us to show more if the system $(U, \mathrm{cl})$ is semiregular (see [3]). A system is semiregular if no closed set can be written as the union of a pair of its proper closed subsets. In a semiregular system we showed in [3] there 
exist supermaximal closed subsets in each r.e. nonzero Turing and dependence degree $\delta$, where $M \in L(U)$ is supermaximal if $\operatorname{dim}(U / M)=\infty$ and for all $Q \in L(U)$ with $Q \supset M$ either $Q=U$ or $\operatorname{dim}(Q / M)<\infty$. We can modify the construction in [3] to ensure the result is "mitotic".

Proposition 1.13. Let $(U, \mathrm{cl})$ be semiregular with C.I.P. Let $\delta$ be an r.e. nonrecursive degree. Then $U$ contains a maximal pair $(A, B)$ such that $d(D(A))=$ $d(A)=d(D(B))=d(B)=d(A+B)=d(D(A+B))=\delta$.

Proof. In [3] we showed that $(U, \mathrm{cl})$ is semiregular if and only if, for all $A, B \subset U$ and $C \in L(U)$ with $A \cup B$ independent over $C$ and $A \cap B=\varnothing$, there exists $\eta$ such that

$$
\eta \in \mathrm{cl}_{C}(A \cup B)-\mathrm{cl}_{C}(A)-\operatorname{cl}_{C}(B)
$$

(see [3], Proposition 2.4). Let $a_{0}, \ldots, a_{n}, \ldots$ be a recursive basis for $U$ and $W_{0}, W_{1}, \ldots$ an effective enumeration of the r.e. closed subsets of $U$.

We construct $I=\cup_{s} I_{s}, V=\cup_{s} V_{s}, A=\cup_{s} A_{s}, B=\cup_{s} B_{s}, I^{A}=\cup_{s} I_{s}^{A}$ and $I^{B}=\cup_{s} I_{s}^{B}$ in stages so that $V=\operatorname{cl}(A \cup B), A=\operatorname{cl}\left(I^{A}\right), B=\operatorname{cl}\left(I^{B}\right), I^{A} \cap I^{B}=$ $\varnothing, A$ and $B$ and independent closed sets, $I^{A} \cup I^{B}$ is a basis for $V$ and $V$ is supermaximal. We use Yates permitting and coding to control the degrees of $A, B, I^{A}, I^{B}$ and $V$ (see [17]).

At each stage $s$ let $b_{0}^{s}<b_{1}^{s}<\cdots$ list in order a cobasis for $V_{s}$ at stage $s$. Let $\operatorname{supp}_{s}(x)$ denote the support of $x$ at stage $s$ relative to $I_{s}^{A} \cup I_{s}^{B} \cup\left\{b_{0}^{s}, b_{1}^{s}, \ldots\right\}$. Define

$$
g(s, x)= \begin{cases}-1 & \text { if } x \in \operatorname{cl}\left(I_{s}^{A} \cup I_{s}^{B}\right), \\ n & \text { if } n \text { is the greatest number such that } b_{n}^{s} \in \operatorname{supp}_{s}(x) .\end{cases}
$$

Let $\boldsymbol{\delta} \neq \mathbf{0}$ be an r.e. degree, $f$ a 1-1 (total) recursive function with $f(\omega)=Z$ and $Z \equiv_{T} \delta$. Let $\langle\cdot, \cdot\rangle$ denote the standard pairing function $\langle\cdot, \cdot\rangle: \omega \times \omega \rightarrow \omega$.

Requirements.

$$
\begin{aligned}
& Q^{A}: I^{A} \equiv_{T} d(A) \equiv_{T} d(D(A)) \equiv_{T} \delta, \\
& Q^{B}: I^{B} \equiv_{T} d(B) \equiv_{T} d(D(B)) \equiv_{T} \delta, \\
& R^{A \cup B}: A \text { and } B \text { are independent complements. }
\end{aligned}
$$

For each $n \in \omega$ and $e \in \omega$

$$
\begin{aligned}
& P_{\langle e, n\rangle}: \text { If } W_{e} \supset V \text { and } \operatorname{dim}\left(W_{e} / U\right)+\infty \text { then } a_{n} \in W_{e} . \\
& N_{e}: \lim _{s} b_{e}^{s}=b_{e} \text { exists. }
\end{aligned}
$$


Definition 1.13.1. $P_{\langle e, n\rangle}$ requires attention at stage $s+1$ if

(i) $a_{n} \notin \mathrm{cl}\left(W_{e}^{s+1} \cup V_{s}\right)$,

(ii) $\exists x \in W_{e}^{s+1}$ such that $\left\{x, a_{n}\right\}$ is independent over $V_{s},\left\{b_{0}^{s}, \ldots, b_{\langle e, n\rangle}^{s}, x\right\}$ is independent over $V_{s}$ and

$$
x \notin \mathrm{cl}_{V_{s}}\left(b_{0}^{s}, \ldots, b_{\langle e, n\rangle}^{s}, a_{n}\right),
$$

such that $\exists \eta \in \operatorname{cl}_{V_{s}}\left(x, a_{n}\right)-\operatorname{cl}_{V_{s}}\left(a_{n}\right)$ such that

$$
g(\eta, s) \geqslant \max \{f(s),\langle e, n\rangle+1\} .
$$

(iii) $\langle e, n\rangle$ is least with respect to (i), (ii).

Construction. Stage 0. Set $I_{0}=I_{0}^{A}=I_{B}^{0}=\varnothing, A_{0}=B_{0}=V_{0}=\operatorname{cl}(\varnothing)$ and $b_{i}^{0}=$ $a_{i}$ for all $i \in \omega$.

Stage $s+1$. If no $P_{\langle e, n\rangle}$ requires attention for $\langle e, n\rangle \leqslant s+1$ set $I_{s+1}^{A}=I_{s}^{A} \cup$ $\left\{b_{f(s)}^{s}\right\}$ and $I_{s+1}^{B}=I_{s}^{B} \cup\left\{b_{f(s)+1}^{s}\right\}$.

$$
\text { Set } b_{i}^{s+1}= \begin{cases}b_{i}^{s} & \text { if } i<f(s), \\ b_{i+2}^{s} & \text { if } i \geqslant f(s) .\end{cases}
$$

If $P_{\langle e, n\rangle}$ requires attention with $x$ least for $\langle e, n\rangle$ and $\eta$ least for $x$ there are 3 cases (a), (b) and (c) below. and

Case (a). $g(\eta, s)=f(s)$. Set $I_{s+1}^{A}=I_{s}^{A} \cup\left\{\eta, b_{f(s)+1}^{s}\right\}, I_{s+1}^{B}=I_{s}^{B} \cup\left\{b_{f(s)+2}^{s}\right\}$

$$
b_{i}^{s+1}= \begin{cases}b_{i}^{s} & \text { for } i<f(s), \\ b_{i+3}^{s} & \text { for } i \geqslant f(s) .\end{cases}
$$

Case (b). $g(\eta, s)=f(s)+1$. Set $I_{s+1}^{A}=I_{s}^{A} \cup\left\{\eta, b_{f(s)}^{s}\right\}$ and $I_{s+1}^{B}=I_{s}^{B} \cup$ $\left\{b_{f(s)+2}^{s}\right\}$ and

$$
b_{i}^{s+1}= \begin{cases}b_{i}^{s} & \text { for } i<f(s), \\ b_{i+3}^{s} & \text { for } i \geqslant f(s) .\end{cases}
$$
and

Case (c). $g(\eta, s)>f(s)+1$. Set $I_{s+1}^{A}=I_{s}^{A} \cup\left\{\eta, b_{f(s)}^{s}\right\}, I_{s+1}^{B}=I_{s}^{B} \cup\left\{b_{f(s)+1}^{s}\right\}$

$$
b_{i}^{s+1}= \begin{cases}b_{i}^{s} & \text { for } i<f(s), \\ b_{i+2}^{s} & \text { for } f(s) \leqslant i<g(\eta, s) \\ b_{i+3}^{s} & \text { for } i \geqslant g(\eta, s)\end{cases}
$$

In all of the above cases set $I_{s+1}=I_{s+1}^{A} \cup I_{s+1}^{B}, A_{s+1}=\operatorname{cl}\left(I_{s+1}^{A}\right), B_{s+1}=$ $\operatorname{cl}\left(I_{s+1}^{B}\right)$ and $V_{s+1}=\operatorname{cl}\left(I_{s+1}\right)$.

End of construction. 
The lemmata which need to be checked are:

LEMMA 1.13.2. $I=\cup_{s} I_{s}$ is independent.

LemMA 1.13.3. Each $P_{\langle e, n\rangle}$ requires attention at most once.

Lemma 1.13.4. All the $N_{e}$ are met.

Lemma 1.13.5. All the $P_{\langle e, n\rangle}$ are met.

LEMMA 1.13.6. $d(I)=d(D(I))=d\left(I^{A}\right)=d\left(D\left(I^{A}\right)\right)=d\left(I^{B}\right)=d\left(D\left(I^{B}\right)\right)$ $\geqslant \delta$.

Lemma 1.13.7. $\left\{b_{0}, b_{1}, \ldots\right\} \equiv_{T} I \leqslant_{T} \delta$.

As the proofs of the above lemmas with the exception of 1.13 .6 are similar to those in [3] we do not prove them here. The proof of 1.13.6 is similar to [3] Lemma 3.13 with the added twist that we need to ensure the degrees of the splitting of $I$ into $I^{A}$ and $I^{B}$ are also of Turing (and dependence) degree greater than or equal to $\delta$. This is similar to 1.14 .5 below and the proof is left to the reader.

We can also give a simpler direct construction of a maximal pair. It has the added advantage that it is more widely applicable than the construction of 1.13 , in particular, it applies to systems without semiregularity. In particular, this and the remaining results constitute an analysis of the class of effectively nowhere simple subsets of $\omega$.

Proposition 1.14. Let $\delta \neq \mathbf{0}$ be an r.e. nonzero degree. Then $(U, \mathrm{cl})$ contains an r.e. maximal pair $(A, B)$ such that $\delta=d(A)=d(D(A))=d(B)=d(D(B))$ with $A$ and $B$ independent.

Proof. We build $A=\cup_{s} A_{s}$ and $B=\cup_{s} B_{s}$ in stages so that $(A, B)$ are the desired maximal pair. To ensure $A_{T} \geqslant \delta$ and $B_{T} \geqslant \delta$ as we did in 1.13 we ensure that at each stage either $b_{f(s)}^{s}$ or $b_{f(s)+1}^{s}$ is put into $A_{s}$ and either $B_{f(s)+1}^{s}$ or $b_{f(s)+2}^{s}$ is put into $B_{s}$. As in 1.13 we use Yate's permitting to ensure $A \leqslant_{T} \delta$ and $B \leqslant_{T} \delta$. We retain the notions of $\operatorname{supp}_{s}(x)$ and $g(x, s)$ that we had in 1.13. We also retain the basis $\left\{a_{i} \mid i \in \omega\right\}$ and cobasis $\left\{b_{i}^{s} \mid i \in \omega\right\}$ of $U$ over $\operatorname{cl}\left(A_{s} \cup B_{s}\right)$.

Our requirements are simply

$$
P_{e}^{A}: W_{e} \supset A \& \operatorname{dim}\left(W_{e} / A\right)=\infty \quad \text { implies } W_{e} \cap B \neq \operatorname{cl}(\varnothing)
$$




$$
\begin{aligned}
& P_{e}^{B}: W_{e} \supset B \& \operatorname{dim}\left(W_{e} / B\right)=\infty \quad \text { implies } W_{e} \cap A \neq \operatorname{cl}(\varnothing) \\
& N_{e}: \lim _{s} b_{e}^{s}=b_{e} \quad \text { exists } \\
& Q: A \equiv_{T} B \equiv_{T} A \cup B \equiv_{T} \delta \equiv_{T} D(B) \equiv_{T} D(A) \\
& R^{A \cup B}: A \text { and } B \text { are independent. }
\end{aligned}
$$

The priority ranking is:

$$
Q, R^{A \cup B}, N_{0}, P_{0}^{A}, P_{0}^{B}, P_{1}^{A}, P_{1}^{B}, \ldots
$$

DEFINITION 1.14.1. $P_{e}^{A}$ requires attention at stage $s+1$ if

(i) $\operatorname{cl}\left(W_{e}^{s+1}\right) \cap B^{s}=\operatorname{cl}(\varnothing)$,

(ii) $3 \theta \in \operatorname{cl}\left(W_{e}^{s+1}\right)$ such that $g(s, \theta) \geqslant \max \{e, f(s)\}$,

(iii) $P_{e}^{A}$ has highest priority satisfying (i), (ii) above, or (i), (ii) above with $B$ in place of $A$. (Of course, here we define $P_{e}^{B}$ to require attention similarly with $B$ in place of $A$ and $A$ in place of $B$.)

At each stage $s$ we construct bases $I_{A}^{s}$ for $A_{s}$ and $I_{B}^{s}$ for $B_{s}$.

Construction. Stage 0 . Set $b_{i}^{0}=a_{i}$ for all $i \in \omega$ where $T=\left\{a_{i} \mid i \in \omega\right\}$ is an recursive basis for $U$ with $a_{i} \geqslant i$ for all $i \in \omega$. Set $A^{0}=B^{0}=\operatorname{cl}\left(I_{A}^{0}\right)$ where $I_{A}^{0}=I_{B}^{0}=\varnothing$.

Stage $s+1$. If no $P_{e}^{A}$ or $P_{e}^{B}$ requires attention set $I_{A}^{s+1}=I_{A}^{s} \cup\left\{b_{f(s)}^{s}\right\}, I_{B}^{s+1}=$ $I_{B}^{s} \cup\left\{b_{f(s)+1}^{s}\right\}$ and $A_{s+1}=\operatorname{cl}\left(I_{A}^{s+1}\right), B_{s+1}=\operatorname{cl}\left(I_{B}^{s+1}\right)$ and

$$
b_{i}^{s+1}= \begin{cases}b_{i}^{s} & \text { if } i<f(s), \\ b_{s+2}^{s} & \text { if } i \geqslant f(s) .\end{cases}
$$

If $P_{e}^{A}$ requires attention and $\theta$ is least for $e$ there are 3 cases:

Case (i). $g(s, \theta)=f(s)$. Set $I_{B}^{s+1}=I_{B}^{s+1} \cup\{\theta\} \cup\left\{b_{f(s)+2}^{s}\right\}, \quad I_{A}^{s+1}=I_{A}^{s} \cup$ $\left\{b_{f(s)+1}^{s}\right\}$ and

$$
b_{i}^{s+1}= \begin{cases}b_{i}^{s} & \text { if } i<f(s), \\ b_{i+3}^{s} & \text { if } i \geqslant f(s) .\end{cases}
$$

Case (ii). $g(s, \theta)=f(s)+1$. Set $I_{B}^{s+1}=I_{B}^{s} \cup\{\theta\} \cup\left\{b_{f(s)+2}^{s}\right\}$, and $I_{A}^{s+1}=I_{A}^{s}$ $\cup\left\{b_{f(s)}^{s}\right\}$.

$$
b_{i}^{s+1}= \begin{cases}b_{i}^{s} & \text { if } i<f(s), \\ b_{i+3}^{s} & \text { if } i \geqslant f(s) .\end{cases}
$$


Case (iii). $g(s, \theta)>f(s)+1$. Set $I_{B}^{s+1}=I_{B}^{s} \cup\{\theta\} \cup\left\{b_{f(s)+1}^{s}\right\}, I_{A}^{s+1}=I_{A}^{s} \cup$ $\left\{b_{f(s)}^{s}\right\}$ and set

$$
b_{i}^{s+1}= \begin{cases}b_{i}^{s} & \text { if } i<f(s) \\ b_{i+2}^{s} & \text { if } f(s) \leqslant i<g(s, \theta) \\ b_{i+3}^{s} & \text { if } i \geqslant g(s, \theta)\end{cases}
$$

In cases (i), (ii) and (iii) set $A^{s+1}=\operatorname{cl}\left(I_{A}^{s+1}\right)$ and $B^{s+1}=\operatorname{cl}\left(I_{B}^{s+1}\right)$.

Use a similar construction if $P_{e}^{B}$ requires attention. Let $I_{A}=\cup_{s} I_{A}^{s}, I_{B}=\cup_{s} I_{B}^{s}$, $A=\operatorname{cl}\left(I_{A}\right)$ and $B=\operatorname{cl}\left(I_{B}\right)$.

End of construction.

The following lemma is easily proved by induction (see, for example, [3] for details).

LemMa 1.14.2. $I_{A} \cap I_{B}=\varnothing$ and $I_{A} \cup I_{B}$ is independent.

LEMMA 1.14.3. $\lim _{s} b_{e}^{s}=b_{e}$ exists.

Proof. The $b_{e}^{s}$ only change when $e \geqslant f(s)$ and this can happen at most finitely often.

Note also the $P_{e}^{A}$ and $P_{e}^{B}$ require attention at most once.

Lemma 1.14.4. All the $P_{e}^{A}$ and $P_{e}^{B}$ are met.

Proof. Let $P_{e}^{A}$ be the requirement of highest priority not met. Let $t_{0}$ be stage where all the earlier requirements that ever require attention do so before stage $t_{0}$ and, for all $i \leqslant e, b_{i}^{t_{0}}=b_{i}$. Now the hypotheses are that $\operatorname{dim}\left(W_{e} / A\right)=\infty$, $W_{e} \supset A$ and $W_{e} \cap B=\operatorname{cl}(\varnothing)$. We show $f(\omega)=A$ is recursive, giving a contradiction.

Let $z \in \omega$. Let $z^{\prime}=\max \left\{b_{e}, z+1\right\}$. We can find a stage $t_{z}>t_{0}$ such that $\exists \theta \in W_{e}^{t}$ with $g\left(t_{z}, \theta\right)>z^{\prime}+1\left(\right.$ as $\left.\operatorname{dim}\left(W_{e} / A\right)=\infty\right)$. It follows that, as $P_{e}^{A}$ can never require attention,

$$
g\left(t_{z}, \theta\right)<f\left(t_{z}\right) .
$$

By the priority scheme, and by construction the $b_{i}^{s}$ can only change when $i>f(s)$. It follows that $\forall s>t_{z}\left(g\left(t_{z}, \theta\right)<f(s)\right)$. Therefore $\forall s>t_{z}(z<f(s))$ and so

$$
z \in f(\omega) \leftarrow z \in(f(\omega))^{t_{z}} .
$$

Let $R=\left\{b_{0}, b_{1}, \ldots\right\}$. 
LEMMA 1.14.5. $R \equiv_{T} I_{B} \cup I_{A T} \geqslant \delta$.

Proof. Let $z \in \omega$. Recursively in $I_{A} \cup I_{B}$ we can find a stage $t_{z}$ where

$$
b_{i}^{t_{z}}=b_{i} \quad \text { all } i \leqslant z .
$$

It follows that $\forall s>t_{z}(f(s)>z)$. Thus $I_{A} \cup I_{B T} \geqslant f(\omega)$ and $\operatorname{deg}(f(\omega))=\delta$.

LEMMA 1.14.6. $f(\omega)=Z_{T} \geqslant I_{A} \cup I_{B} \equiv_{T} R$.

Proof. Let $z \in \omega$. Recursively in $Z$ we can find a stage $t_{z}$ such that

$$
\forall s>t_{z}(f(s)>z+2) .
$$

Then $z \in I_{A} \cup I_{B} \leftarrow z \in\left(I_{A} \cup I_{B}\right)^{t_{2}}$.

Thus $R \equiv_{T} I_{A} \cup I_{B} \equiv_{T} Z$ and $\operatorname{deg}(Z)=\delta$. Trivially $I_{A} \leqslant_{T} I_{A} \cup I_{B}$ and $I_{B} \leqslant_{T} I_{A}$ $\cup I_{B}$. We claim $I_{A} T \geqslant I_{A} \cup I_{B}$. To show this it suffices to show that $\operatorname{deg}\left(I_{A}\right) \geqslant R$. Recursively in $I_{A}$ we can decide whether or not any member $A_{i}$ of $T$ is in $I_{A}$ or not. Now at each stage $s$ either $b_{f(s)}$ or $b_{f(s)+1}$ is put into $I_{A}$. Thus at each stage $s$ a member of $T$ is put into $I_{A}$. Let $a_{i} \in T$. Recursively in $I_{A}$ we can find a stage $s_{i}$ such that

$$
\forall s>s_{i}\left(\psi \in I_{A}^{s}-I_{A}^{s_{i}} \rightarrow \psi>a_{i}\right) .
$$

Therefore $a_{i} \in R \leftrightarrow a_{i} \in R^{s_{i}}$ and so $I_{A T} \geqslant R$. But as we observed earlier $R \equiv_{T} Z$ $=f(\omega)$ and so $\operatorname{deg}\left(I_{A}\right) \geqslant \delta$. Therefore $\operatorname{deg}\left(I_{A}\right)=\operatorname{deg}\left(I_{B}\right)=\operatorname{deg}(R)=\delta$. The reader can check that as we can find $\operatorname{supp}_{I_{A} \cup I_{B} \cup R}(\theta)$ for $\theta \in U$ that we have

$$
d(R)=d\left(I_{A}\right)=d\left(I_{B}\right)=d(B)=d(A)=d(B)=d(D(A))=d(D(B)) .
$$

One observation concerning the above construction is that it works in more general settings than for Steinitz systems with C.I.P. For example, it works for $V_{\infty}(F)$ with affine dependence, that is, $\left(V_{\infty}, \mathrm{K} 1\right)$. We can characterize the closed sets with decompositions into (nowhere simple) maximal pairs. Recall that an r.e. $V$ in $L(U)$ is simple if, for all $Q$ in $L(U)$ with $Q \cap V=\operatorname{cl}(\varnothing), \operatorname{dim}(Q)$ is finite.

Proposition 1.15. Let $Q \in L(U)$. Then $Q$ possesses a decomposition into a maximal pair $Q_{1}, Q_{2}$ if and only if $Q$ is simple.

Proof. $(\rightarrow)$ is obvious.

$(\leftarrow)$ Let $Q$ be simple. Use precisely the same construction as given in Proposition 1.4. Suppose $W_{e} \supset Q_{1}$ and $\operatorname{dim}\left(W_{e} / Q_{1}\right)=\infty$ and $W_{e} \cap Q_{2}=\operatorname{cl}(\varnothing)$, then it follows that $\operatorname{dim}\left(W_{e} / Q\right)=\infty$ and, by construction, $Z=\{x \mid \exists s, t(t>s \& x \in$ $\left.\left.\operatorname{cl}\left(W_{e}^{s}\right) \& x \notin \operatorname{cl}\left(Q^{s}\right) \& x \in\left(Q^{t}\right)\right)\right\}$ is finite (for otherwise we could meet the $P_{\langle e, n\rangle}^{i}$ and so $\operatorname{dim}\left(W_{e} \cap Q_{2}\right)=\infty$, a contradiction). 
Thus, as we showed in Proposition 1.4, this allows us to construct an r.e. set $R$ independent over $\operatorname{cl}(Q)=Q$ (in the notation of 1.4). Thus by C.I.P. $\operatorname{cl}(R) \cap$ $\operatorname{cl}(Q)=\operatorname{cl}(\varnothing)$. It follows that $Q$ is not simple-a contradiction.

For systems with C.I.P. there is an easy way to produce simple closed sets. Let $H$ be a hypersimple subset of degree $\delta$ of a recursive basis $B$ of $U$. Then $\operatorname{cl}(H)$ is simple of Turing and dependence degrees $\delta$. The reader may supply the details (see Remmel [10]). This allows us to show:

COROLlaRY 1.16. Let $\delta \neq 0$ be an r.e. degree. Then there exists an r.e. closed set $Q$ with both its Turing and dependence degrees $\delta$ such that there exists an r.e. closed set $Q^{\prime}$ with $\left(Q, Q^{\prime}\right)$ a maximal pair. In particular, each r.e. degree contains an effectively nowhere simple closed set.

Proof. Left to reader.

We close this paper with a number of open questions. In more general systems (that is without C.I.P.) it is still possible to define a notion of nowhere simplicity. We do this by using a method similar to that used in [4] for simplicity but keeping everything in terms of "dimension over".

Definition 1.17. Let $S \in L(U)$ with $\operatorname{dim}(U / S)=\infty$. We say $S$ is (*)-nowhere simple if, whenever $Q \in L(U)$ with $\operatorname{dim}(Q / S)=\infty$, there exists $Q^{\prime} \subset Q$ such that $Q^{\prime} \cap S=\operatorname{cl}(\varnothing), Q^{\prime} L(U)$ and $\operatorname{dim}\left(\operatorname{cl}_{S}\left(Q^{\prime}\right) / S\right)=\infty$.

It is not clear whether or not there is an analogue of Proposition 1.4 for general Steinitz systems in terms of (*)-nowhere simplicity. In fact this is not even known for $\left(F_{\infty}, \mathrm{cl}\right)$. It is known that many properties of subsets of recursive bases can be lifted to properties of the closed subsets they generate. For example Shore (in [7]) showed that a maximal subset of a recursive basis generates a maximal closed subset. In [4] it is shown that a similar result holds for major subsets and major closed subsets. Does a nowhere simple subset of a recursive basis generate a nowhere simple closed subset of $U$ ? Shore [14] showed that no creative set is nowhere simple. To do this he showed one creative set is not nowhere simple and then used Myhill's uniqueness theorem (see for example [14] for details). In our setting we cannot do this. In [7], Metakides and Nerode defined a creative subspace and showed that the analogue of Myhill's Theorem simply does not work for $L\left(V_{\infty}\right)$. Thus we may ask if either a creative subspace (closed subset) or an $E U H$ kernel (see [7]) can be nowhere simple. Another open problem is the following: let $R$ be a recursive basis and $(A, B)$ a maximal pair in $L(R)$, is $(\mathrm{cl}(A), \mathrm{cl}(B))$ a maximal pair in $(U, \mathrm{cl})$ ? 
Added in proof. Whilst the paper was in proof I became aware of A. Nerode and J. B. Remmel's paper "Recursion theory on matroids," 41-67 in Patras Logic Symposion, North-Holland (Studies in Logic 109), 1982. There the authors independently analyzed nowhere simplicity in matroids, and obtained many of the results presented here. Also they show that the analogues of Soare's automorphism base results hold for $V_{\infty}$, but not as an analogue of [14] as I had thought.

\section{References}

[0] C. J. Ash and R. Downey, 'Decidable subspaces and recursively enumerable subspaces', to appear.

[1] J. T. Baldwin, 'Recursion theory and abstract dependence', to appear.

[2] J. N. Crossley and A. Nerode, 'Recursive equivalence on matroids', Aspects of effective algebra ed. J. N. Crossley ( $\forall$ Book Company, Steel's Creek, Australia, 1981).

[3] R. Downey, 'Abstract dependence and recursion theory', Monash Logic paper No. 33.

[4] R. Downey, 'On a question of Retzlaff', Z. Math. Logik Grundlagen Math., to appear.

[5] R. Downey, 'On the lattice of r.e. filters, axiomatizable theories and $\pi_{1}^{0}$ classes', submitted.

[6] R. Downey, 'On bases of supermaximal subspaces and Steinitz systems', in preparation.

[7] G. Metakides and A. Nerode, 'Recursively enumerable vector spaces', Ann. Math. Logic 11 (1977) 147-171.

[8] G. Metakides and A. Nerode, 'Recursion theory on fields and abstract dependence', J. Algebra 65 (1980) 36-59.

[9] A. Nerode and R. Smith, 'The undecidability of the lattice of recursively enumerable subspaces', Proceedings of the Third Brazilion Conference on Mathematical Logic, pp. 245-252, eds. Arruda, Da Costa, Sette (1982).

[10] J. B. Remmel, 'Recursion theory on algebraic structures with independent sets', Ann. Math. Logic 18 (1980) 153-191.

[11] A. Retzlaff, 'Direct summands of r.e. vector spaces', Z. Math. Logik Grundlagen Math. 25 (1979) 363-372.

[12] H. J. Rogers, Jr., Theory of recursive functions and effective computability (McGraw-Hill, New York, 1967).

[13] R. Shore, 'Determining automorphism of recursively enumerable sets', Proc. Amer. Math. Soc. 65 (1977), 318-326.

[14] R. Shore, 'Nowhere simple sets and r.e. sets', J. Symbolic Logic 43 (1978), 513-530.

[15] R. Shore, 'Controlling the dependence degree of a recursively enumerable vector space', $J$. Symbolic Logic 43 (1978), 13-23.

[16] R. Shore, 'The infinite injury priority method', J. Symbolic Logic 41 (1976), 513-530.

[17] C. E. M. Yates, 'Three theorems on the degrees of recursively enumerable sets', Duke Math. J. 32 (1965), 461-468.

Department of Mathematics

National University of Singapore

Kent Ridge, 0511

Singapore 